\title{
COVID-19 Vaccine Response in People with Multiple Sclerosis
}

\author{
Emma C. Tallantyre, MRCP, PhD $10,{ }^{1,2 \dagger}$ Nicola Vickaryous, $\mathrm{PhD}^{3+}$ Valerie Anderson, $\mathrm{PhD},{ }^{1}$ \\ Aliye Nazli Asardag, BSc, ${ }^{4}$ David Baker, $\mathrm{PhD} \odot{ }^{4}{ }^{4}$ Jonathan Bestwick, $\mathrm{MSc}_{1}{ }^{3}$ \\ Kath Bramhall, BSc, ${ }^{5}$ Randy Chance, $\mathrm{MSc}^{4,6}$ Nikos Evangelou, FRCP, PhD, \\ Katila George, BSc, ${ }^{3}$ Gavin Giovannoni, PhD, FRCP, $3,4,8$ Andrew Godkin, PhD, FRCP, 9,10 \\ Leanne Grant, BSc, Katharine E. Harding, MRCP, PhD, ${ }^{11}$ Aimee Hibbert, BSc, \\ Gillian Ingram, MRCP, PhD, ${ }^{12}$ Meleri Jones, $\mathrm{PhD}^{4}{ }^{4}$ Angray S. Kang, $\mathrm{PhD},{ }^{4,6}$ \\ Samantha Loveless, PhD, ${ }^{1}$ Stuart J. Moat, PhD, ${ }^{13,14}$ Neil P. Robertson, FRCP, PhD, ${ }^{1,2}$ \\ Klaus Schmierer, FRCP, PhD $\oplus^{4,8}$ Martin J. Scurr, PhD, ${ }^{9,15}$ Sita Navin Shah, MBBS, ${ }^{3}$ \\ Jessica Simmons, ${ }^{1}$ Matthew Upcott, BSc, ${ }^{1}$ Mark Willis, MRCP, MD, ${ }^{2}$ \\ Stephen Jolles, FRCP, FRCPath, PhD, ${ }^{5,9 \ddagger}$ and Ruth Dobson, FRCP, PhD $\oplus^{3,8 \ddagger}$
}

Objective: The purpose of this study was to investigate the effect of disease modifying therapies on immune response to severe acute respiratory syndrome-coronavirus 2 (SARS-CoV-2) vaccines in people with multiple sclerosis (MS). Methods: Four hundred seventy-three people with MS provided one or more dried blood spot samples. Information about coronavirus disease 2019 (COVID-19) and vaccine history, medical, and drug history were extracted from questionnaires and medical records. Dried blood spots were eluted and tested for antibodies to SARS-CoV-2. Antibody titers were partitioned into tertiles with people on no disease modifying therapy as a reference. We calculated the odds ratio of seroconversion (univariate logistic regression) and compared quantitative vaccine response (Kruskal Wallis) following the SARS-CoV-2 vaccine according to disease modifying therapy. We used regression modeling to explore the effect of vaccine timing, treatment duration, age, vaccine type, and lymphocyte count on vaccine response.

View this article online at wileyonlinelibrary.com. DOI: 10.1002/ana.26251

Received Aug 12, 2021, and in revised form Oct 18, 2021. Accepted for publication Oct 18, 2021.

Address correspondence to Professor Stephen Jolles, Immunodeficiency Centre for Wales, University Hospital of Wales, Cardiff, UK. E-mail: jollessr@cardiff.ac.uk Dr Ruth Dobson. Preventive Neurology Unit, Wolfson Institute of Population Health, Queen Mary University London, London, UK. E-mail: ruth.dobson@qmul.ac.uk

†These authors contributed equally and are joint first authors.

These authors contributed equally and are joint corresponding authors.

From the ${ }^{1}$ Division of Psychological Medicine and Clinical Neuroscience, School of Medicine, Cardiff University, Cardiff, UK; ${ }^{2}$ Department of Neurology, University Hospital of Wales, Cardiff, UK; ${ }^{3}$ Preventive Neurology Unit, Wolfson Institute of Population Health, Queen Mary University London, London, UK; ${ }^{4}$ Blizard Institute, Barts and the London School of Medicine and Dentistry, Queen Mary University of London, London, UK; ${ }^{5}$ mmunodeficiency Centre for Wales, University Hospital of Wales, Cardiff, UK; ${ }^{6}$ Centre for Oral Immunobiology and Regenerative Medicine, Barts and the London School of Medicine and Dentistry, Queen Mary University of London, London, UK; ${ }^{7}$ Department of Clinical Neurology, University of Nottingham, Nottingham, UK;

${ }^{8}$ Department of Neurology, Barts Health NHS Trust, London, UK; ${ }^{9}$ Division of Infection and Immunity, School of Medicine, Cardiff University, Cardiff, UK;

${ }^{10}$ Department of Gastroenterology and Hepatology, University Hospital of Wales, Cardiff, UK; ${ }^{11}$ Department of Neurology, Royal Gwent Hospital, Newport, UK; ${ }^{12}$ Department of Neurology, Morriston Hospital, Swansea, UK; ${ }^{13}$ Wales Newborn Screening Laboratory, Department of Medical Biochemistry, Immunology and Toxicology, University Hospital of Wales, Cardiff, UK; ${ }^{14}$ School of Medicine, Cardiff University, Cardiff, UK; and ${ }^{15}$ ImmunoServ Ltd., Cardiff, UK

Additional supporting information can be found in the online version of this article.

(C) 2021 The Authors. Annals of Neurology published by Wiley Periodicals LLC on behalf of American Neurological Association. 1 This is an open access article under the terms of the Creative Commons Attribution-NonCommercial License, which permits use, distribution and reproduction in any medium, provided the original work is properly cited and is not used for commercial purposes. 
Results: Compared to no disease modifying therapy, the use of anti-CD20 monoclonal antibodies (odds ratio $=0.03$, $95 \%$ confidence interval $[\mathrm{Cl}]=0.01-0.06, p<0.001$ ) and fingolimod (odds ratio $=0.04 ; 95 \% \mathrm{Cl}=0.01-0.12$ ) were associated with lower seroconversion following the SARS-CoV-2 vaccine. All other drugs did not differ significantly from the untreated cohort. Both time since last anti-CD20 treatment and total time on treatment were significantly associated with the response to the vaccination. The vaccine type significantly predicted seroconversion, but not in those on anti-CD20 medications. Preliminary data on cellular T-cell immunity showed $40 \%$ of seronegative subjects had measurable anti-SARS-CoV-2 T cell responses.

Interpretation: Some disease modifying therapies convey risk of attenuated serological response to SARS-CoV-2 vaccination in people with MS. We provide recommendations for the practical management of this patient group.

ANN NEUROL 2021;00:1-12

ndividuals with inflammatory neurological disorders have faced considerable uncertainty during the coronavirus disease 2019 (COVID-19) pandemic, related to both the potential risks posed by their underlying medical disorder, and the immunomodulatory treatment needed to manage their disease. Factors that appear to confer increased vulnerability for poor outcomes following symptomatic COVID-19 include advanced disability, obesity, male sex, and the use of immunosuppressive drugs, in particular, anti-CD20 monoclonal antibodies. ${ }^{1}$ As a result, people with chronic neurological disease in several countries were advised to strictly self-isolate during periods of high community transmission.

The development of effective vaccines against COVID-19 infection, which are safe for use in vulnerable groups, offer hope for a reduction in COVID-19 related mortality and morbidity. However, uncertainties remain over vaccine efficacy for those taking immunosuppressive and/or immunomodulatory drugs, including multiple sclerosis (MS) disease modifying therapies (DMTs). Previous studies have demonstrated a blunted vaccine response in people with MS receiving ocrelizumab ${ }^{2}$ and fingolimod ${ }^{3}$; however, vaccine responses to a truly novel biological antigen (ie, one giving rise to human disease, rather than keyhole limpet hemocyanin $[\mathrm{KLH}]$ ) have only been subject to limited study. Data are urgently required on the efficacy of COVID-19 vaccination for those individuals receiving immunomodulating drugs to guide them regarding risks related to reopening policies, and to inform policy around booster vaccinations ${ }^{4}$ or additional strategies for those who are unable to respond adequately to the vaccination. There may be the potential to optimize vaccination response in people receiving MS DMTs, for example, by temporarily suspending continuous DMT or extending the interval between intermittent DMTs. However, adopting this approach in the absence of supportive evidence needs to be balanced against unnecessary and potentially disabling MS disease activity.

The study of MS treatment offers a relatively unique opportunity. People with MS tend to remain on a continuous platform (ie, non-induction) of DMTs for many years, rather than having treatment "holidays" or breaks, due to the irreversible nature of disease-related disability. In addition, DMTs are given as monotherapy, rather than in combination, as is practice in some other autoimmune diseases and selected hematological malignancies. Furthermore, untreated people with MS are generally considered to have a "normal" immune response to vaccination. As a result, response to vaccination in people with MS offers an opportunity to understand the role of individual medications, and their mode(s) of action on the development of an adequate vaccine response.

In this study, we investigate the effect of MS DMTs on the serological response to the COVID-19 vaccination in a large multicenter cohort. Remotely patient-collected, posted dried blood spots were used to obtain samples from people with MS for antibody evaluation following vaccination. By establishing the antibody response to COVID-19 vaccines in relation to patient, treatment, and vaccine characteristics we aim to better inform future guidance for people with MS on vaccination policy, DMT management, and infection protection.

\section{Methods}

People with MS from 5 UK MS centers (Cardiff, Newport, Nottingham, Royal London Hospital [Barts Health NHS Trust], and Swansea) were invited to participate in this study. Samples were analyzed in 2 laboratories: the University Hospital of Wales (UHW), Cardiff and the Queen Mary University of London (QMUL).

\section{Participants}

Potential participants under the care of 5 MS clinics were approached either in person at the time of scheduled appointments, in writing by email or letter, or by advertisement, asking them to provide one or more dried blood spot samples along with a questionnaire, providing data on isolation behavior, employment setting, clinical or laboratory-confirmed cases of COVID-19 infection, vaccine dates, and vaccine type. Potential participants were approached between November 2020 and June 2021, including people with MS on all DMTs, regardless of their decision regarding vaccination. Where participants 
agreed to take part, they were provided with a kit for selfsampling. Participants also provided consent for access to their medical records by study staff, allowing verification of MS diagnosis, and details of DMT prescription, including date of initiation and most recent administration. Hospital records were also used to confirm all cases of COVID-19 requiring hospital admission, and to extract laboratory results, such as total lymphocyte counts from routine blood monitoring data. All participants provided written informed consent to take part in this study. This study has Research Ethics Committee approval (REC 20/SW/0104 and 20/NE/0176).

\section{Sample Collection}

Dried blood spot sampling was undertaken remotely, avoiding the need for phlebotomist time and hospital visits by potentially immunosuppressed patients. A kit containing instructions on how to collect samples, lancets to obtain capillary blood, a blood spot collection device (PerkinElmer 226 Spot Saver or Whatman 903 Protein Saver card), and a return mail envelope was posted to participants at their home address. Participants were asked to date their dried blood spot test card and return it using first class surface mail as soon as possible. Samples received at the UHW were stored desiccated at $-20^{\circ} \mathrm{C}$ until analysis; those received at QMUL were stored at room temperature for a maximum of a week prior to spot punching and elution, with subsequent storage at $-20^{\circ} \mathrm{C}$.

$\mathrm{T}$ cell responses to SARS-CoV-2 in 16 participants with negative humoral response to SARS-CoV-2 were measured using a commercially available whole blood assay (ImmunoServ Ltd.), as previously described. ${ }^{5}$ Briefly, $10 \mathrm{ml}$ venous blood from each patient was collected into sodium heparin vacutainers (BD) and processed in the laboratory within 6 hours of blood draw.

\section{Antibody Testing for SARS-CoV-2 in Dried Blood Spot Specimens}

Dried blood spots were used to determine serological status in two laboratories; 376 in UHW and 97 in QMUL.

In UHW, samples were analyzed with the COVIDSeroKlir 2-step enzyme-linked immunosorbent assay (ELISA; Kantaro Biosciences, USA - supplied by EKF Diagnostics, UK), which detects SARS-CoV-2 specific IgG antibodies against the receptor-binding domain $(\mathrm{RBD})^{6,7}$ using resuspended dried blood spots, using previously validated methods. ${ }^{8}$ A 6 mm diameter sub-punch was taken from each dried blood spot using a DELFIA dried blood spot Puncher (PerkinElmer) and placed into $2 \mathrm{ml}$ 96-well plates (Waters \#186002482) containing $600 \mu \mathrm{l}$ of the Kantaro Kit sample diluent (equivalent to
1:100 dilution recommended for the Kantaro kit). The 96-well plates were then covered and shaken at room temperature for 1 hour (as we have shown previously this to be the optimum time to ensure complete extraction of the blood from the filter paper collection device). The plates were then stored at $4^{\circ} \mathrm{C}$ until analysis (usually within 72 hours), although extracted samples were stable for at least 1 week. One hundred microliters of the dried blood spot eluate were transferred to the 96-well ELISA plate and analyzed on an automated platform (DSX; Dynex Technologies, USA) according to the manufacturer's instructions.

The Kantaro assay relies on an assay-specific calibrator to report the ratio of the specimen absorbance to the calibrator absorbance to calculate a cutoff index value. The antibody level in plasma / serum is reported as positive $(\geq 0.7)$ or negative $(<0.7)$. In a series of paired plasma and dried blood spots $(\mathrm{n}=22$ antibody negative and $\mathrm{n}=25$ antibody positive samples), we observed good agreement between paired specimens $(r=0.994)$ and the gradient of the slope was $1.241 \mathrm{x}$. Based upon the negative bias observed in dried blood spot versus paired plasma samples, we calculated the following cutoff index for dried blood spot specimens - positive $(\geq 0.56)$ and negative $(<0.56)$. Quality controls (QCs) as supplied by the manufacturer and in-house prepared dried blood spot QCs (positive and negative) were analyzed on each assay plate.

At QMUL, samples were analyzed using the Globody technique, ${ }^{9}$ with the RBD as the target antigen. A $4 \mathrm{~mm}$ blood spot punch was eluted in $200 \mu \mathrm{l}$ of $1 \%$ Triton X-100 in phosphate-buffered saline (PBS) at room temperature overnight. Eluted samples were assayed the next day or stored at $-20^{\circ} \mathrm{C}$ until analysis. Assays were carried out using $20 \mu \mathrm{l}$ of dried blood spot eluate mixed with $30 \mu \mathrm{l}$ of a $50 \%$ slurry of Protein G agarose equilibrated in PBS (Thermo Fisher Scientific), made up to a final volume of $1.2 \mathrm{ml}$ with $0.05 \%$ PBS-Tween 20 (PBST) and left for 30 minutes on a rotating wheel at room temperature. A PBST wash was performed by pelleting the agarose at $200 \times g$ for 1 minute, aspirating supernatant down to $\sim 100 \mu \mathrm{l}$ and washing with $1,100 \mu \mathrm{l}$ PBST. After washing, an additional $200 \times g$ for 1 minute spin was performed and aspiration of non-agarose bound mixture to $\sim 100 \mu$ l. RBD-GloBody preparation $(8.7 \mu \mathrm{l})$ of $1 \times 10^{8}$ lux units was added to each sample and then made up to $750 \mu \mathrm{l}$ with PBST and left on rotating wheel for 30 minutes, after which the agarose resin was applied to a Pierce $0.8 \mathrm{ml}$ centrifuge column (Thermo Fisher Scientific). Columns were washed 5 times with $750 \mu \mathrm{PBST}$ to remove unbound RBD-GloBody and followed by a final wash with $750 \mu \mathrm{l}$ PBS. Bound IgG and RBD-GloBody in complex with IgG were eluted from spin column with the 
addition of $100 \mu \mathrm{l}$ of $0.1 \mathrm{M}$ Glycine, $\mathrm{pH} 2.7$, and neutralized with $12 \mu \mathrm{l} 0.1 \mathrm{M}$ Tris $\mathrm{pH} 9.0$. Assays on $30 \mu \mathrm{l}$ of the resulting eluate were performed in triplicate using furimazine substrate $(20 \mu \mathrm{l}$ furimazine in $1 \mathrm{ml} 0.1 \%$ bovine serum albumin [BSA] in PBS; Promega). Luminescence was measured on a CLARIOstar plate reader. A limit of blank (LoB) was determined by LoB = mean luminescence:blank +2.58 (standard deviation:blank), values greater than this suggest seroconversion with a confidence of $99 \%$.

\section{Detection of SARS-CoV-2-Specific T Cell Responses}

Whole blood samples were aliquoted into microcentrifuge tubes (Thermo Fisher Scientific) containing pre-aliquoted peptides spanning covering the entire spike (S1 and S2) protein (S), nucleocapsid phosphoprotein (NP), and membrane glycoprotein (M). Additional tubes containing phytohemagglutinin-L (Sigma) or nothing were run alongside as positive and negative controls, respectively. Whole blood samples were incubated at $37^{\circ} \mathrm{C}$ for 20 to 24 hours.
Plasma was harvested from the top of each blood sample the amount of IFN- $\gamma$ in each sample quantified. A previously defined positive SARS-CoV-2-specific $\mathrm{T}$ cell response has a cutoff value of $>23.55 \mathrm{pg} / \mathrm{ml} \mathrm{IFN- \gamma}$ and $50 \%$ above the negative (unstimulated) control value, differentiating naïve controls from prior COVID-19 vaccination and/or previously infected subjects (sensitivity of $93.6 \%$ and specificity of $80.8 \%) .^{5}$

\section{Statistical Analysis}

Participants were categorized according to DMT exposure status at the time of the first COVID-19 vaccination. People were considered exposed if they had received alemtuzumab or cladribine within 4 years, ocrelizumab within 12 months, natalizumab within 8 weeks, fingolimod, dimethyl fumarate, teriflunomide, glatiramer acetate, or beta-interferon within 4 weeks of their vaccine first dose. All other participants were categorized as unexposed to DMT.

Fishers exact test was used to compare the chance of seroconversion following the first and second vaccine dose across different DMTs. Univariate logistic regression with

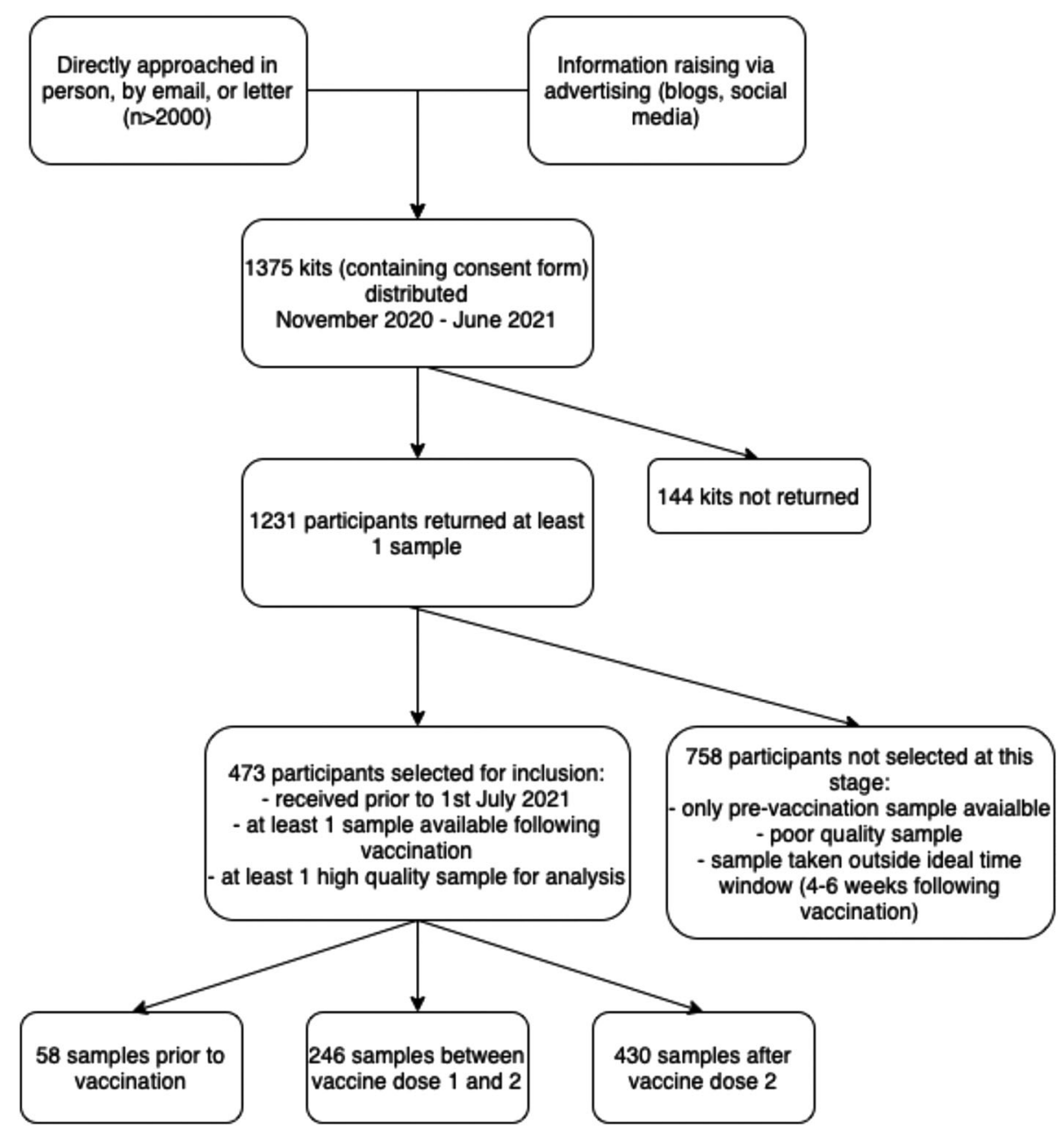

FIGURE 1: Flow chart illustrating recruitment and selection of the study cohort. 
TABLE 1. Vaccine Response According to DMT

\begin{tabular}{|c|c|c|c|c|c|}
\hline & $\begin{array}{l}\text { Serostatus } \\
\text { following dose } 1 \\
\text { (positive:negative, } \\
\% \text { seroconverted) }\end{array}$ & $\begin{array}{l}\text { Serostatus } \\
\text { following dose } 2 \\
\text { (positive:negative, } \\
\% \text { seroconverted) }\end{array}$ & $\begin{array}{l}\text { OR seroconversion } \\
\text { following dose } 2 \\
\text { (OR; } 95 \% \mathrm{CI})\end{array}$ & 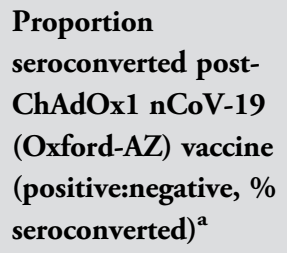 & $\begin{array}{l}\text { Proportion } \\
\text { seroconverted post- } \\
\text { BNT162b2 (Pfizer) } \\
\text { vaccine (positive: } \\
\text { negative, } \% \\
\text { seroconverted) }^{b}\end{array}$ \\
\hline All participants & 140:106 (57\%) & $280: 150(65 \%)$ & NA & $107: 80(57 \%)^{\mathrm{c}}$ & $113: 37(75 \%)^{\mathrm{c}}$ \\
\hline No DMT & $77: 18(81 \%)$ & $85: 7(92 \%)$ & 1 (reference) & $27: 2(93 \%)$ & 41:0 (100\%) \\
\hline Anti-CD20 mAb ${ }^{d}$ & $7: 45(13 \%)$ & $33: 101(25 \%)$ & $0.03(0.01-0.06)^{\mathrm{e}}$ & $16: 57(22 \%)$ & $11: 24(31 \%)$ \\
\hline Natalizumab & 23:8 (74\%) & $56: 5(92 \%)$ & $0.92(0.28-3.05)$ & 28:2 (93\%) & $25: 2(93 \%)$ \\
\hline Alemtuzumab & 14:4 (78\%) & $24: 4(86 \%)$ & $0.49(0.13-1.83)$ & $14: 3(82 \%)$ & $9: 1(90 \%)$ \\
\hline Dimethyl fumarate & $9: 7(56 \%)$ & $35: 3(92 \%)$ & $0.96(0.23-3.93)$ & $12: 3(80 \%)$ & $14: 0(100 \%)$ \\
\hline Cladribine & $1: 7(13 \%)$ & $16: 4(80 \%)$ & $0.33(0.09-1.26)$ & $5: 3(63 \%)$ & $9: 1(90 \%)$ \\
\hline Glatiramer acetate & 2:0 (100\%) & $3: 0(100 \%)$ & $N A^{f}$ & $N A^{g}$ & $N A^{g}$ \\
\hline Fingolimod & $4: 15(21 \%)$ & $12: 24(33 \%)$ & $0.04(0.01-0.12)^{\mathrm{e}}$ & $5: 10(33 \%)$ & $4: 9(31 \%)$ \\
\hline Interferon beta & 3:0 (100\%) & $5: 1(83 \%)$ & $0.41(0.04-4.03)$ & $N A^{\mathrm{g}}$ & $N A^{g}$ \\
\hline Teriflunomide & $0: 2(0 \%)$ & $3: 0(100 \%)$ & $N A^{f}$ & $\mathrm{NA}^{\mathrm{g}}$ & $N A^{g}$ \\
\hline & $p<0.0001$ & \multicolumn{4}{|l|}{$p<0.0001$} \\
\hline \multicolumn{6}{|c|}{ 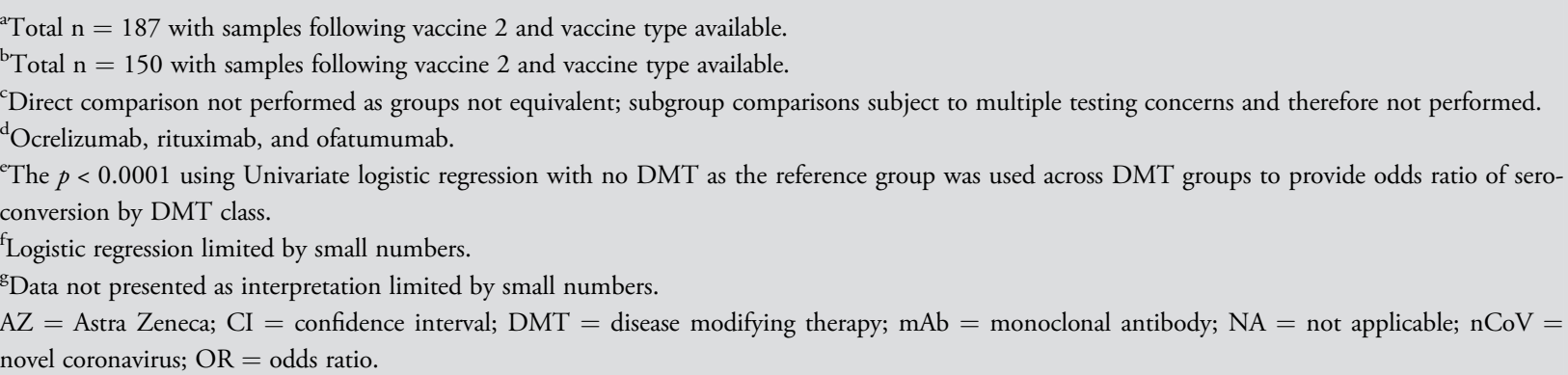 } \\
\hline
\end{tabular}

no DMT as the reference group was used across DMT groups to provide odds ratio (OR) of seroconversion by DMT class. Due to the different laboratory approaches used to determine antibody titer, quantitative vaccine responses from each laboratory were partitioned into tertiles with no DMT as the reference group. Kruskal Wallis test was used to compare the proportion of vaccine responses in each tertile between DMT following the second vaccine.

The impact of time between last dose of anti-CD20 monoclonal antibody (ocrelizumab and rituximab, excluding ofatumumab given different administration schedule) and first vaccine dose, and odds of seroconversion, was established using univariate logistic regression. Similar analyses were performed in order to establish the impact of time since treatment initiation on odds of seroconversion. Linear regression across tertiles with the same outputs was performed in order to explore the impact on quantitative vaccine response.

Finally, stepwise multivariate logistic regression was used to model the likelihood of a positive antibody response to COVID-19 vaccine measured after the second vaccine dose. Two groups of interest were selected for the study - (1) those either not on DMT, or taking DMT shown not to influence seroconversion in the univariate analyses, and (2) those who had been treated with antiCD20 monoclonal antibodies. Samples from people taking fingolimod were excluded from these analyses. An initial model, including gender and vaccine type, was generated; subsequently age, Expanded Disability Status Scale (EDSS; grouped into 0.0-4.0, 4.5-5.5, 6.0-6.5, and 7.0-10.0), previous COVID-19 symptoms, time between vaccine doses, time from second vaccine to sampling, total 


\begin{tabular}{|c|c|c|c|}
\hline & \multicolumn{2}{|c|}{$\begin{array}{l}\text { Serostatus } \\
\text { following dose } \\
2 \text { (positive:negative, } \\
\% \text { seroconverted) }\end{array}$} & \multirow{2}{*}{$\begin{array}{l}\text { Difference } \\
\text { between } \\
\text { centers }(p)\end{array}$} \\
\hline & Cardiff & QMUL & \\
\hline No DMT & $84: 5(94 \%)$ & $2: 1(66 \%)$ & NS \\
\hline Anti-CD20 mAb ${ }^{a}$ & $25: 59(30 \%)$ & $8: 42(16 \%)$ & NS \\
\hline Natalizumab & $48: 2(96 \%)$ & $8: 3(73 \%)$ & 0.04 \\
\hline Alemtuzumab & 22:1 (96\%) & $3: 2(60 \%)$ & NS \\
\hline Dimethyl fumarate & $27: 2(93 \%)$ & $8: 1(89 \%)$ & NS \\
\hline Cladribine & $8: 1(89 \%)$ & $8: 3(73 \%)$ & NS \\
\hline Glatiramer acetate & $3: 0(100 \%)$ & NA & - \\
\hline Fingolimod & $12: 21(36 \%)$ & $0: 3(0 \%)$ & NS \\
\hline Interferon beta & $5: 1(83 \%)$ & NA & - \\
\hline Teriflunomide & $3: 0(100 \%)$ & NA & - \\
\hline \multicolumn{4}{|c|}{$\begin{array}{l}{ }^{a} \text { Ocrelizumab, rituximab, and ofatumumab. } \\
\text { DMT = disease modifying therapy; mAb }=\text { monoclonal antibody; } \\
\text { NA = not applicable; NS = not significant; QMUL = Queen Mary } \\
\text { University of London. }\end{array}$} \\
\hline
\end{tabular}

time on most recent DMT, and lymphocyte count were added to the model in a stepwise manner. Only those factors shown to significantly improve model fit were retained in the final model. Mann-Whitney $U$ test was used to compare potential influence on anti-SARS-CoV-2 IgG titer according to vaccine type.

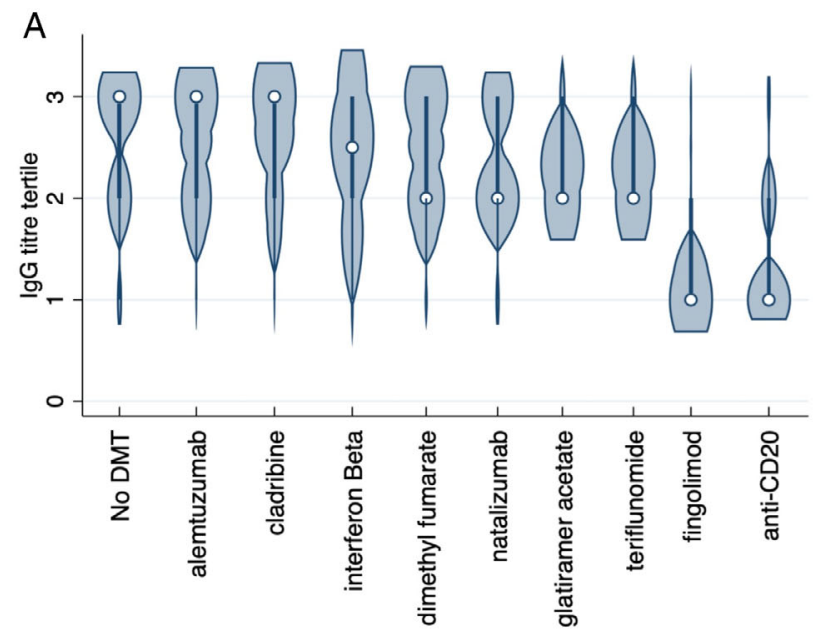

Statistical analysis was performed in Stata version 16 (Stat Corp. Ltd.).

\section{Results}

One thousand three hundred seventy-five kits were sent out across all sites between November 2020 and June 2021. A total of 1,231 (89.5\%) participants returned at least one sample (Fig 1). Participants returning at least one dried blood spot sample following vaccination were considered for inclusion in this analysis based on time of sample post-vaccination (aiming for 4-8 weeks post-vaccination) and quality of sample provided.

Of the 473 participants eligible for inclusion in this analysis, 58 participants provided samples prior to vaccination, 246 during the interval between their first and second vaccine doses, and 430 following the second vaccine dose. Demographic and clinical features are given in Supplementary Table S1, and are provided by DMT in Supplementary Table S2. Participants not receiving DMT were, on average, older and more disabled than those on DMT (see Supplementary Table S2). Those with an unknown vaccination type (or no vaccination) were more likely to have missing EDSS.

Participants received their initial vaccine dose between December 8, 2020, and June 9, 2021. Vaccine type was available in 404 (85.4\%) participants; 180 reported vaccinations with BNT162b2 (Pfizer-BioNTech) mRNA vaccine, and 224 reported vaccinations with adenoviral vector vaccine (223 participants received ChAdOx1 nCoV-19 [OxfordAstraZeneca], and one received Johnson \& Johnson). One participant reported initial vaccination with the ChAdOx1 nCoV-19 (Oxford-AstraZeneca) adenoviral vector vaccine with the second vaccine dose BNT162b2 mRNA (Pfizer-

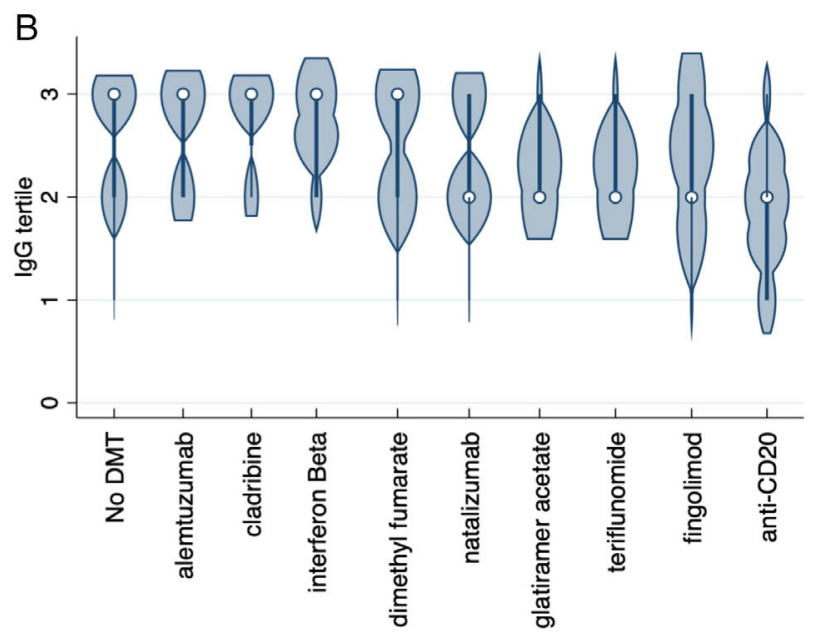

FIGURE 2: Violin plot illustrating anti-SARS-CoV-2 antibody response following complete COVID-19 vaccine course, according to DMT. The plot shows distribution of results according to tertiles, using the "no DMT" group as a reference. Circle indicates median, bold line indicates interquartile range. (A) All samples (B) seropositive samples only. COVID-19= coronavirus disease 2019; DMT = disease modifying therapy; SARS-CoV-2 = severe acute respiratory syndrome-coronavirus 2. 
BioNTech); this participant was excluded from analyses using vaccine type as a variable. Median interval between vaccine doses was 10.0 weeks (range $=3.0-14.0$ weeks, interquartile range $[\mathrm{IQR}]=6.1-11.0$ weeks).

\section{Pre-Vaccine Serology}

Of the 58 vaccine naïve baseline samples, 6 (10.3\%) were seropositive and $52(89.7 \%)$ seronegative for SARS-CoV2. Of the 6 participants who were seropositive at baseline; 4 were on DMT ( 1 was on glatiramer acetate, 1 was on alemtuzumab [year 1 only; April 2019], and 2 were on ocrelizumab). Data on prior symptoms suggestive of COVID-19 was available for 44 out of 58 participants, including all 6 who were seropositive at baseline. Only 3 out of 6 seropositive participants reported prior symptoms or prior positive SARS-CoV-2 polymerase chain reaction (PCR). Eleven (19.2\%) of the 52 individuals who were seronegative at baseline self-reported prior symptoms suggestive of COVID-19 (of whom 3 had confirmatory SARS-CoV-2 PCR). There was no significant association between patient-reported historical COVID-19 infection and baseline seropositivity.

\section{Post-Vaccine Serology}

Overall, 140 of 246 (56.9\%) participants with a sample available between the first and second vaccine doses demonstrated a positive $\operatorname{IgG}$ response (Table 1), and 280 of $430(65.1 \%)$ at $>4$ weeks post second dose. Univariate logistic regression demonstrated that, compared to no DMT, the use of anti-CD20 monoclonal antibodies ( $\mathrm{OR}=0.03,95 \%$ confidence interval $[\mathrm{CI}]=0.01-0.06, p<0.001)$ and fingolimod $(\mathrm{OR}=0.04,95 \% \mathrm{CI}=0.01-0.12)$ were associated with lower seroconversion following vaccine 2 (see Table 1). All other DMTs did not differ significantly from the untreated cohort. Subgroup analysis between the UHW and QMUL analyzed cohorts did not demonstrate any significant difference between the response to complete vaccination course in any DMT that passed the multiple testing threshold (Table 2).

One-way analysis of variance (ANOVA) to examine the effect of DMT on antibody titer was limited by ceiling

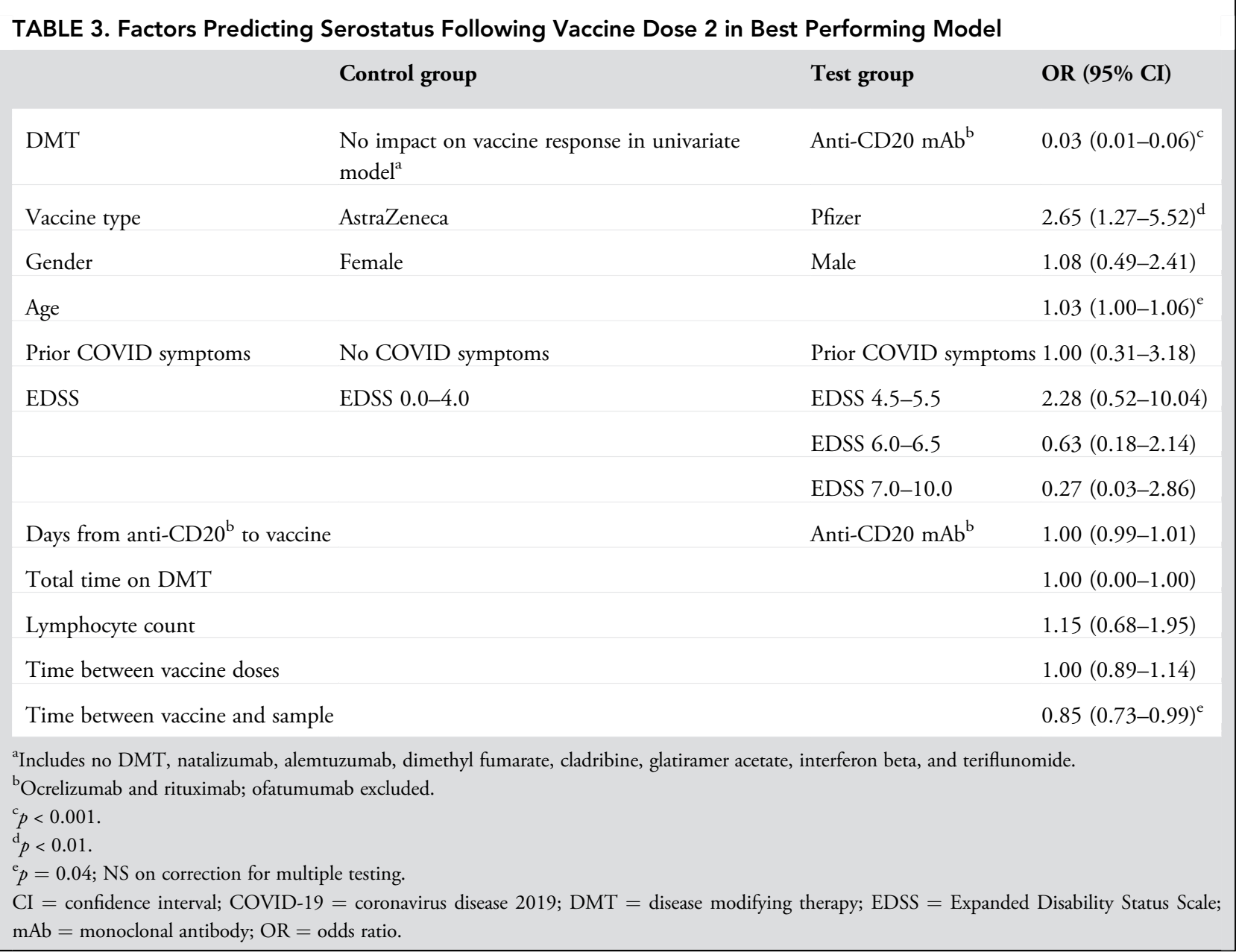



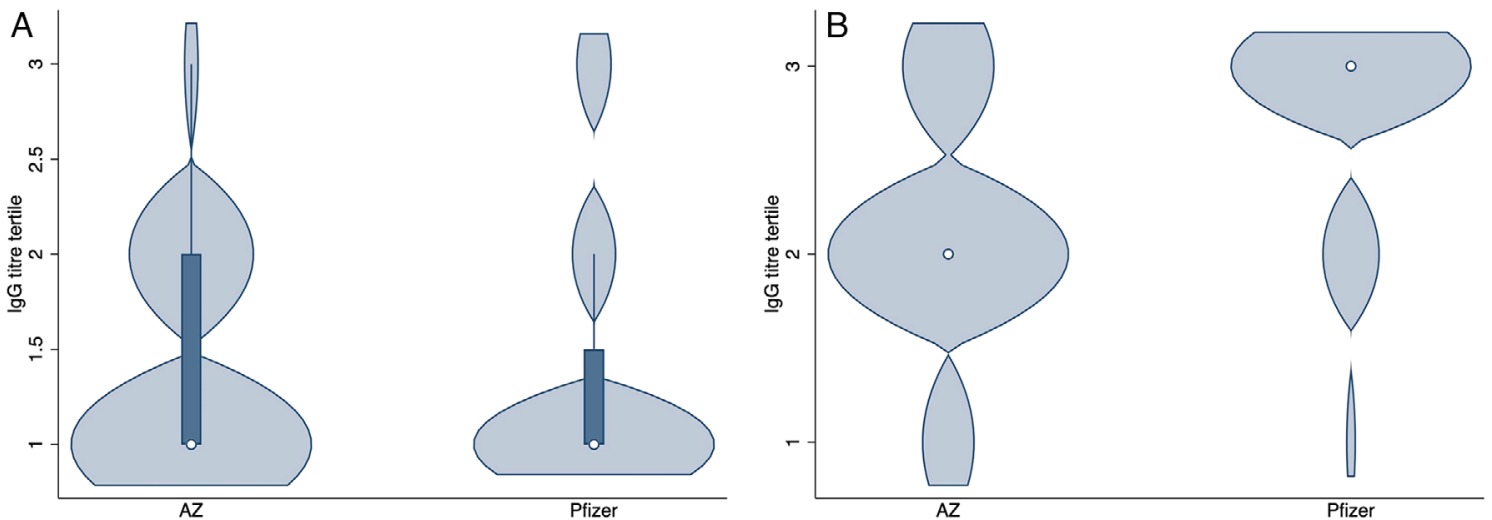

FIGURE 3: Violin plot illustrating anti-SARS-CoV2 antibody response following complete COVID-19 vaccine course, according to vaccine type. The plot shows distribution of results according to tertiles, using the "no DMT" group as a reference. The circle indicates median, and the bold line indicates interquartile range. Astra Zeneca: ChAdOx1 nCoV-19 (Oxford-AstraZeneca), Pfizer: BNT162b2 mRNA (PfizerBioNTech) vaccine. (a) People who had received anti-CD20 monoclonal antibodies. (b) All other DMT (fingolimod excluded). COVID-19 = coronavirus disease 2019; DMT = disease modifying therapy; $\mathrm{nCov}=$ novel coronavirus; SARS-CoV-2 = severe acute respiratory syndrome-coronavirus 2.

effects in the UHW cohort and small numbers in treatment groups when restricting to the QMUL cohort. Using tertiles defined by antibody titers in the untreated cohort, Kruskal Wallis equality of populations rank test demonstrated a significant difference between populations in IgG titer tertile between DMT (Fig 2). This persisted

\begin{tabular}{|c|c|c|c|c|c|c|c|}
\hline Participant & DMTs & $\begin{array}{l}\text { Time since DMT } \\
\text { start }(\mathbf{m o})^{\mathrm{a}}\end{array}$ & Age & Vaccination type & T-cell result ${ }^{\mathbf{b}}$ & $\begin{array}{l}\text { IFN-gamma } \\
\text { response }\end{array}$ & $\begin{array}{l}\text { Negative } \\
\text { control value }\end{array}$ \\
\hline 1 & Alemtuzumab & 5 & 42 & AstraZeneca & Positive & 26.0 & 6.9 \\
\hline 2 & Fingolimod & 85 & 38 & AstraZeneca & Negative & 41.0 & 34.9 \\
\hline 3 & Fingolimod & 95 & 39 & Pfizer & Negative & 25.2 & 19.0 \\
\hline 4 & Fingolimod & 53 & 50 & Pfizer & Negative & 30.1 & 29.6 \\
\hline 5 & Fingolimod & 86 & 50 & Pfizer & Negative & 8.2 & $<7.8$ \\
\hline 6 & Fingolimod & 24 & 51 & AstraZeneca & Negative & 22.9 & 20.1 \\
\hline 7 & Fingolimod & 76 & 43 & AstraZeneca & Positive & 46.5 & $<7.8$ \\
\hline 8 & None & N/a & 59 & AstraZeneca & Positive & 244.9 & $<7.8$ \\
\hline 9 & Ocrelizumab & 23 & 48 & Pfizer & Negative & 14.7 & $<7.8$ \\
\hline 10 & Ocrelizumab & 20 & 58 & Pfizer & Negative & $<7.8$ & $<7.8$ \\
\hline 11 & Ocrelizumab & 21 & 26 & Pfizer & Positive & 236.5 & 13.0 \\
\hline 12 & Ocrelizumab & 9 & 48 & Pfizer & Positive & 38.3 & $<7.8$ \\
\hline 13 & Ocrelizumab & 21 & 50 & Pfizer & Positive & 49.7 & $<7.8$ \\
\hline 14 & Ocrelizumab & 22 & 56 & Pfizer & Positive & 731.5 & 16.8 \\
\hline 15 & Rituximab & 58 & 41 & AstraZeneca & Negative & 51.6 & 34.7 \\
\hline 16 & Rituximab & 133 & 57 & AstraZeneca & Negative & 35.5 & 31.4 \\
\hline
\end{tabular}

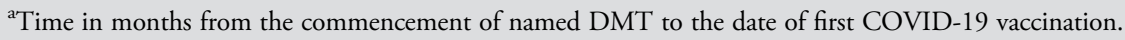

${ }^{\mathrm{b}}$ A positive SARS-CoV-2-specific T cell response was defined as an IFN-gamma response $>23.55 \mathrm{pg} / \mathrm{ml}$ and $50 \%$ above the negative (unstimulated) control value, as previously determined. ${ }^{5}$

COVID-19 = coronavirus disease 2019; DMT = disease modifying therapy; SARS-CoV-2 = severe acute respiratory syndrome-coronavirus 2. 
regardless of whether both seropositive and seronegative samples were included in the analysis (Fig 2A, $p=0.0001$ ), or if the analysis was restricted to only the seropositive cohort (Fig 2B, $p=0.0022$ ).

Neither time since the last anti-CD20 treatment nor the total time on anti-CD20 DMT predicted (binary) serostatus after the second vaccine, however, the small number of seropositive individuals limited the power in this analysis. Five of 72 people treated with anti-CD20 therapies within the 5 months (150 days) prior to the initial vaccination mounted a response following the second vaccine dose, compared to 5 of 9 people treated $\geqq 7$ months (210 days) prior to initial vaccination $(p=0.037$, Fishers exact test). Linear regression across tertiles, excluding a single outlier, was performed in the anti-CD20 cohort. In this analysis, both time from last treatment administration to initial vaccination $(p=0.033)$ and total time on treatment $(p=0.025)$ demonstrated a significant relationship with serological response to vaccination.

DMT type was then dichotomized into antiCD20 monoclonal antibody (ocrelizumab, rituximab, and ofatumumab) and control MS with normal vaccine response (no DMT, natalizumab, alemtuzumab, dimethyl fumarate, cladribine, glatiramer acetate, interferon beta, and teriflunomide). These DMTs all demonstrated a statistically similar serological response to vaccination. Samples from fingolimod treated individuals were excluded from this analysis. In order to develop a multivariate regression model, a stepwise multivariate regression model was developed. Factors retained in the final, best performing model were vaccine type and treatment (anti-CD20 vs others). Although the time between administration of the second vaccine dose and age showed significance on initial testing, they did not pass the threshold for multiple testing on Bonferroni correction $(p=0.005$; Table 3). Overall $\operatorname{IgG}$ levels were not included in this analysis as only 4 of 473 participants had levels below the lower limit of normal. Finally, the impact of vaccine type on SARS-CoV-2-IgG titer was examined across DMT groups (anti-CD20 vs others). There was no difference between the serological response to the vaccination according to vaccine type in those who had received anti-CD20 monoclonal antibodies (Fig 3A; $p=0.39$ ). However, in the control group, those who were vaccinated with the BNT162b2 mRNA (Pfizer-BioNTech) vaccine had a significantly greater IgG response to vaccination than those vaccinations with the ChAdOx1 nCoV-19 (Oxford-AstraZeneca) vaccine (Fig $3 \mathrm{~B} ; p<0.0001$ ).

\section{T-Cell Response}

The absence of a serological response to vaccination may seem explicable when B cell (anti-CD20) targeted therapies are used. The T-cell response to SARS-CoV2 was tested in 16 participants, all of whom had negative humoral response after a full course of COVID-19 vaccination. A positive T-cell response was observed in $40 \%$ of these subjects: 1 participant who received alemtuzumab 5 months earlier, 1 participant not receiving DMT, 1 of 6 people receiving fingolimod, 4 of 6 people receiving ocrelizumab, and 0 of 2 people receiving rituximab (Table 4).

\section{Discussion}

The COVID-19 pandemic may transition to endemic infection, but the consequences are likely to continue for years to come. The rapid development of vaccines offers hope for a reduction in illness severity and viral transmission, with consequent lifting of restrictions. This study answers some of the questions that have arisen around vaccine efficacy in people with MS taking immunomodulatory drugs. With a cohort of almost 500 patients, we have demonstrated that both DMT type and vaccine type affect humoral immune response to the COVID-19 vaccination, offering an opportunity to provide advice about infection-prevention and control for people at risk. However, certain DMTs, including some with high MS treatment efficacy, appear to have no effect on the response to the COVID-19 vaccination.

Our results align with a previous, smaller study, ${ }^{10}$ showing attenuated response to the BNT162b2 mRNA (Pfizer-BioNTech) vaccine in people with MS receiving ocrelizumab and fingolimod. The number of patients in our cohort allows us to interpret results with more certainty, examine the impact of dosing time, and importantly understand the impact of vaccine type. Anti-CD20 monoclonal antibodies were the DMTs most strongly associated with lack of seroconversion following the COVID-19 vaccination. Their high efficacy and relatively favorable safety profile has offered a welcome addition to the MS DMT landscape; however, once ocrelizumab is commenced, rapid and profound B-cell depletion occurs, with few people repopulating B-cells prior to their 6 months interval infusion. ${ }^{11,12}$ These drugs have previously been shown to be associated with attenuated humoral response to recall and novel vaccinations. ${ }^{2}$ This presents an opportunity to test people on anti-CD20 DMTs for evidence of secondary antibody deficiency by routinely measuring Ig classes, and also by measuring functional immunity to vaccines for which normative reference ranges exist, for example, pneumococcus and haemophilus. ${ }^{13}$ The intact $\mathrm{T}$ cell response in 4 of 6 vaccine nonresponders on ocrelizumab provides some potential reassurance to those who have received the vaccine close to their infusion time. 
We also found that fingolimod, a sphingosine1-phospohate (S1P) receptor modulator, is associated with both a substantially attenuated humoral response to the COVID-19 vaccination and a low number of $T$ cell responders in the vaccine nonresponders. This does not reflect the moderately attenuated vaccine responses seen in clinical trials, ${ }^{14}$ but has been previously demonstrated in smaller real-world studies of COVID-19 vaccine response. ${ }^{10}$ Given the substantial MS rebound/reactivation associated with fingolimod cessation, ${ }^{15}$ medication withdrawal to facilitate successful vaccination is associated with significant risk if there is no managed switch to an alternative DMT. This strategy carries additional complexities, including the potential for carryover DMT-associated risk, for example, lymphopenia, and uncertainties over how to manage a switch back if appropriate. It seems likely that the attenuated vaccine response seen with fingolimod is a class effect that will be seen in other S1P products including siponimod and ponesimod; however, the different half-lives of these medications means that drug-specific studies are needed.

Our data offers the opportunity to tailor advice for people with MS who are at risk of attenuated humoral response to vaccination. Our study suggested that delaying commencement of fingolimod or anti-CD20 DMT should be considered in new starters, to allow time for vaccination. Our data did not demonstrate a relationship between delaying established anti-CD20 infusions and improving seroconversion but this analysis is likely to have been underpowered. We did demonstrate a modest (but significant) impact of delaying established anti-CD20 infusions on COVID-19 antibody titer, raising questions over whether substantially extended dosing intervals to allow B-cell recovery might enhance vaccine response. The proportion of individuals who seroconverted increased between vaccine 1 and 2 , in keeping with studies in the healthy control population, ${ }^{16}$ supporting the use of a booster vaccination, particularly for those who may have had an attenuated response to the initial vaccination. However, further study is required to determine whether the booster vaccination induces humoral immunity in all those who failed to mount a humoral response to the first course. It is not yet routine to test anti-SARS-CoV-2 antibodies in clinical practice, but knowledge of seronegative status in people with MS may allow more vigilant infection control precautions, for example, continuing to socially distance and ensuring that household contacts are vaccinated, and may also allow individualized treatment of emergent COVID-19 infection, for example, monoclonal antibodies to SARSCoV-2 have been shown to benefit those who are seronegative. $^{17}$
Our overall finding of a differential humoral response according to vaccine type replicates data in healthy control cohorts, ${ }^{18}$ but was not replicated in people on anti-CD20 DMT. Whereas this provides no current rationale for recommending one vaccine type over another in those at risk of an attenuated response, this is deserving of further study given potential limitations of power. Current evidence suggests that heterologous vaccine regimens may elicit stronger antibody and T-cell responses. ${ }^{19,20}$ The effect of age on vaccine response in this cohort is in line with data from the general population, ${ }^{16}$ and may be entirely due to age-related immunosenescence, ${ }^{18}$ or there may be an additional impact from collider bias related to age, disability progression, and DMT choice.

This study made use of dried blood spot sampling, reducing the need for potentially vulnerable people to attend healthcare facilities during the pandemic as well as reduced costs for phlebotomist time and equipment. Dried blood spot sampling has been used since the 1960s for neonatal screening for inborn errors of metabolism, ${ }^{21}$ but wider medical uses, including serological screening of IgG levels, ${ }^{22,23}$ have proven particularly useful during the COVID-19 pandemic. ${ }^{24}$ Whereas extensive work was undertaken to develop and validate the assays used in this study, the lack of a gold standard for RBD assay development was a potential limitation; because a true negative result was defined based on historical samples. In order to increase the power for this study, samples were analyzed in 2 laboratories and data were pooled. The assay used in the UHW laboratory was subject to significant ceiling effects, with around a third of untreated patients having samples with an IgG titer at or above ceiling. Although subgroup analysis demonstrated similar seroconversion between the 2 assays, and the use of tertiles avoided pooling 2 nonlinear scales, this represents a potential limitation of the study. Reports of vaccine type and dates, as well as prior COVID-19 infection were self-reported in this study, introducing the possibility of inaccuracy. A further caveat is that whereas all DMT exposures were part of monotherapy regimens, some people with MS had been exposed sequentially to several therapies. Given the large number of DMTs, we were not powered to explore the effect of sequential therapies, so it cannot be wholly excluded.

Pre-vaccine samples were only available for 58 of 473 participants in this study, of whom $10 \%$ had serological evidence of prior infection. The lack of full concordance between pre-vaccine serology and history of previous COVID-19 infection is not unexpected and is likely to reflect a combination of asymptomatic infections (those seropositive pre-vaccine), and either unproven COVID-19 (lack of laboratory confirmation) or decline of anti-SARS-CoV-2 antibodies following proven COVID- 
19 (those seronegative at baseline). ${ }^{25}$ It is likely that in the full cohort who provided post-vaccine samples, at least a similar proportion of all participants already had antiSARS-CoV-2 antibodies at baseline. There is evidence of an association between the presence of antibodies to SARS-CoV-2 at the time of vaccination and a greater subsequent immune response to the COVID-19 vaccine. ${ }^{26}$ Given the lack of association between prior reported COVID-19 symptoms and baseline seropositivity in this cohort, we did not feel confident to use this as a marker of prior infection to explore enhanced vaccine response.

SARS-CoV-2 is a strongly immunogenic virus that can induce antigen-specific antibody production in the majority of infected patients. ${ }^{27}$ However, the immune correlates of protection following COVID-19 vaccines have not yet been fully established. Antibody responses to COVID-19 vaccination appear to be protective. ${ }^{28}$ Specifically, anti-SARS-Cov-2 RBD IgG levels appear to correlate with virus neutralization titres, ${ }^{29-31}$ and durable antibody responses have been shown to be associated with more rapid recovery from infection. ${ }^{32}$ In animal models, neutralizing antibody (NAb) levels following vaccination using the spike antigen correlate with protection against SARS-CoV-2, ${ }^{33,34}$ and antibody dependent functional immunity is enhanced by a COVID-19 vaccine. ${ }^{35}$ Although NAbs are frequently considered a key component of the immune response after viral infection, antiviral $\mathrm{T}$ cell mediated immunity is also central to viral clearance. ${ }^{36}$ SARS-CoV-2-specific $\mathrm{CD}^{+}$and $\mathrm{CD}^{+}{ }^{+} \mathrm{T}$ cells have been associated with milder disease in acute and convalescent individuals. ${ }^{37}$ We measured T-cell responses to SARS-CoV-2 in a small subcohort of people with a negative antibody response to COVID-19 vaccines in this study. The preliminary data demonstrated that $40 \%$ of people with negative serological response did nevertheless mount a measurable $\mathrm{T}$-cell response. Ongoing/future work on magnitude and durability of immune response required to protect from COVID-19 is needed in this and other cohorts, including whether a booster vaccination improves the chance of seroconversion in people with attenuated immune response to the initial vaccination, and the relative importance of both humoral and $\mathrm{T}$ cell immunity in terms of symptomatic and severe infection. Crucially, it will be important to monitor the population who are seronegative yet demonstrate strong $\mathrm{T}$ cell responses, to understand how these responses wane over time, and whether these subjects are protected from future SARS-CoV-2 infections.

In conclusion, this study demonstrates the impact of DMT type, vaccine type, and age on the vaccine response. It provides high quality evidence to support advice for people with MS, and indicates routes for future study, including the need for clinical trials to guide advice around balancing risks and potential benefits of suspending or delaying treatment.

\section{Acknowledgments}

The authors would like to acknowledge the help of Swee Vickie Nixon, Ray Wynford-Thomas, Marija Cauchi, Catherine McConnell, Cynthia Butcher, Miranda Maria Piana Parra, Joseph Bishop, and Raees Samli in the running of the study. UHW: Support for equipment and consumables was provided by Cardiff and Vale UHB and Cardiff University. Kantaro Biosciences, LLC, kindly provided quantitative COVID-19 serologic test kits to measure antibody levels for participants in this study. Salary for Samantha Loveless was partly provided by the BRAIN Unit Infrastructure Award (Grant no: UA05). The BRAIN Unit is funded by Welsh Government through Health and Care Research Wales. QMUL: Work performed as part of this study at QMUL is funded by Merck Serono Ltd., Feltham, UK, an affiliate of Merck KGaA with additional support from the MS community via crowdfunding. Assay development was funded by Barts Charity. This work was performed within the PNU, which is funded by Barts Charity.

\section{Author Contributions}

E.C.T., R.D., S.J., N.P.R., N.V., G.G., A.G., K.S., M.S., D.B., M.W., and S.M. contributed to the conception and design of the study. N.V., V.A., A.N.A., J.B., R.C., N.E., K.G., L.G., A.G., K.E.H., A.H., G.I., M.J., A.S.K., S.J.M., S.N.S., M.S., J.S., and S.L. contributed to the acquisition and analysis of data. E.C.T., N.V., R.D., S.J., and N.P.R. contributed to drafting the text or preparing the figures.

\section{Potential Conflicts of Interest}

Biogen, Merck, Novartis, Roche, Sanofi/Genzyme, and Teva all manufacture multiple sclerosis disease modifying therapies that were used in this study, or which could be affected by the study. The following authors have received speaker fees, consultancy fees, and/ or travel expenses to attend educational meetings from one or more of these companies: E.C.T., D.B., R.D., N.E., G.G., A.K., N.P.R., K.S., S.J., M.J.S., and A.G. are co-founders of and holds equity in ImmunoServ Ltd., which provided T-cell analysis. N.V., V.A., A.N.A., J.B., K.B., R.C., K.G., L.G., A.G., K.E.H., A.H., G.I., M.J., A.S.K., S.L., S.J.M., S.N.S., J.S., M.U., and M.W. have no conflicts of interest to declare. 


\section{References}

1. Sormani MP, Salvetti M, Labauge P, et al. DMTs and Covid-19 severity in MS: a pooled analysis from Italy and France. Ann Clin Transl Neurol 2021;8:1738-1744.

2. Bar-Or A, Calkwood JC, Chognot C, et al. Effect of ocrelizumab on vaccine responses in patients with multiple sclerosis: the VELOCE study. Neurology 2020;95:e1999-e2008.

3. Boulton C, Meiser K, David OJ, Schmouder R. Pharmacodynamic effects of steady-state fingolimod on antibody response in healthy volunteers: a 4-week, randomized, placebo-controlled, parallelgroup, multiple-dose study. J Clin Pharmacol 2012;52:1879-1890.

4. Department of Health and Social Care. Most vulnerable could be offered booster COVID-19 vaccines from September. In: GOV.UK [Internet]. 30 Jun 2021. Available at: https://www.gov.uk/ government/news/most-vulnerable-could-be-offered-booster-covid19-vaccines-from-september. Accessed July 132021.

5. Scurr MJ, Zelek WM, Lippiatt G, et al. Whole blood-based measurement of SARS-CoV-2-specific T cell responses reveals asymptomatic infection and vaccine efficacy in healthy subjects and patients with solid organ cancers. medRxiv 2021. https://doi.org/10.1101/2021.06. 02.21258218 .

6. Wajnberg A, Amanat F, Firpo A, et al. Robust neutralizing antibodies to SARS-CoV-2 infection persist for months. Science 2020;370:12271230.

7. Amanat F, Stadlbauer D, Strohmeier S, et al. A serological assay to detect SARS-CoV-2 seroconversion in humans. Nat Med 2020;26: 1033-1036.

8. Moat SJ, Zelek WM, Carne E, et al. Development of a highthroughput SARS-CoV-2 antibody testing pathway using dried blood spot specimens. Ann Clin Biochem 2021;58:123-131.

9. Saxena GK, Theocharopoulos I, Aziz NT, et al. GloBody technology: detecting anti-drug antibody against VH/VL domains. Sci Rep 2020; 10:1860.

10. Achiron A, Mandel M, Dreyer-Alster $S$, et al. Humoral immune response to COVID-19 mRNA vaccine in patients with multiple sclerosis treated with high-efficacy disease-modifying therapies. Ther Adv Neurol Disord 2021;14:17562864211012835.

11. van Lierop ZY, Toorop AA, van Ballegoij WJ, et al. Personalized Bcell tailored dosing of ocrelizumab in patients with multiple sclerosis during the COVID-19 pandemic. Mult Scler 2021;135245852110288. (Epub ahead of print)

12. Baker D, Roberts CAK, Pryce G, et al. COVID-19 vaccine-readiness for anti-CD20-depleting therapy in autoimmune diseases. Clin Exp Immunol 2020;202:149-161.

13. Tallantyre EC, Robertson NP, Jolles S. Secondary antibody deficiency in neurology. Curr Opin Allergy Clin Immunol 2018;18: 481-488

14. Kappos L, Mehling M, Arroyo R, et al. Randomized trial of vaccination in fingolimod-treated patients with multiple sclerosis. Neurology 2015;84:872-879.

15. Barry B, Erwin AA, Stevens J, Tornatore C. Fingolimod rebound: a review of the clinical experience and management considerations. Neurol Ther 2019;8:241-250.

16. Wei J, Stoesser N, Matthews PC, et al. Antibody responses to SARSCoV-2 vaccines in 45,965 adults from the general population of the United Kingdom. Nat Microbiol 2021;6:1140-1149.

17. Group RC, RECOVERY Collaborative Group, Horby PW, Mafham M, et al. Casirivimab and imdevimab in patients admitted to hospital with COVID-19 (RECOVERY): a randomised, controlled, open-label, platform trial. medRxiv. https://doi.org/10.1101/2021.06.15.21258542.

18. Shrotri M, Navaratnam AMD, Nguyen V, et al. Spike-antibody waning after second dose of BNT162b2 or ChAdOx1. Lancet 2021;398: 385-387.
19. Hillus D, Schwarz T, Tober-Lau P, et al. Safety, reactogenicity, and immunogenicity of homologous and heterologous prime-boost immunisation with ChAdOx1-nCoV19 and BNT162b2: a prospective cohort study. medRxiv 2021. https://doi.org/10.1101/2021.05.19. 21257334

20. Barros-Martins J, Hammerschmidt SI, Cossmann A, et al. Humoral and cellular immune response against SARS-CoV-2 variants following heterologous and homologous ChAdOx1 nCoV-19/BNT162b2 vaccination. medRxiv 2021. https://doi.org/10.1101/2021.06.01.21258172

21. Moat SJ, George RS, Carling RS. Use of dried blood spot specimens to monitor patients with inherited metabolic disorders. Int J Neonatal Screen 2020;6:26.

22. Freeman JD, Rosman LM, Ratcliff JD, et al. State of the science in dried blood spots. Clin Chem 2018;64:656-679.

23. Su X, Carlson BF, Wang $X$, et al. Dried blood spots: an evaluation of utility in the field. J Infect Public Health 2018;11:373-376.

24. Roxhed N, Bendes A, Dale M, et al. Multianalyte serology in homesampled blood enables an unbiased assessment of the immune response against SARS-CoV-2. Nat Commun 2021;12:3695.

25. Muecksch F, Wise $H$, Batchelor $B$, et al. Longitudinal serological analysis and neutralizing antibody levels in coronavirus disease 2019 convalescent patients. J Infect Dis 2020;223:389-398.

26. Prendecki M, Clarke C, Brown J, et al. Effect of previous SARSCoV-2 infection on humoral and T-cell responses to single-dose BNT162b2 vaccine. Lancet 2021;397:1178-1181.

27. Deeks JJ, Dinnes J, Takwoingi $Y$, et al. Antibody tests for identification of current and past infection with SARS-CoV-2. Cochrane Database Syst Rev 2020;6:CD013652. https://doi.org/10.1002/14651858. cd013652.

28. Khoury DS, Cromer D, Reynaldi A, et al. Neutralizing antibody levels are highly predictive of immune protection from symptomatic SARSCoV-2 infection. Nat Med 2021;27:1205-1211.

29. Sahin U, Muik A, Derhovanessian E, et al. COVID-19 vaccine BNT162 b1 elicits human antibody and $\mathrm{TH} 1 \mathrm{~T}$ cell responses. Nature 2020;586:594-599.

30. To KK-W, Tsang OT-Y, Leung W-S, et al. Temporal profiles of viral load in posterior oropharyngeal saliva samples and serum antibody responses during infection by SARS-CoV-2: an observational cohort study. Lancet Infect Dis 2020;20:565-574.

31. Ramasamy MN, Minassian AM, Ewer KJ, et al. Safety and immunogenicity of ChAdOx1 $\mathrm{nCoV}-19$ vaccine administered in a prime-boost regimen in young and old adults (COV002): a single-blind, randomised, controlled, phase 2/3 trial. Lancet 2021;396:1979-1993.

32. Chen $Y$, Zuiani A, Fischinger $S$, et al. Quick COVID-19 healers sustain anti-SARS-CoV-2 antibody production. Cell 2020;183:1496-1507.e16.

33. Mercado NB, Zahn $R$, Wegmann $F$, et al. Single-shot Ad26 vaccine protects against SARS-CoV-2 in rhesus macaques. Nature 2020;586: 583-588.

34. Yu J, Tostanoski LH, Peter $L$, et al. DNA vaccine protection against SARS-CoV-2 in rhesus macaques. Science 2020;369:806-811.

35. Barrett JR, Belij-Rammerstorfer S, Dold C, et al. Phase 1/2 trial of SARS-CoV-2 vaccine ChAdOx1 $\mathrm{nCoV}-19$ with a booster dose induces multifunctional antibody responses. Nat Med 2021;27: 279-288.

36. Wyllie $D$, Jones HE, Mulchandani $R$, et al. SARS-CoV-2 responsive $T$ cell numbers and anti-Spike IgG levels are both associated with protection from COVID-19: a prospective cohort study in keyworkers. medRxiv 2021. https://doi.org/10.1101/2020.11.02.20222778

37. Rydyznski Moderbacher C, Ramirez SI, Dan JM, et al. Antigenspecific adaptive immunity to SARS-CoV-2 in acute COVID-19 and associations with age and disease severity. Cell 2020;183:9961012.e19. 